\title{
Doença de Parkinson secundária a COVID-19: uma revisão sistemática
}

\author{
Parkinson's disease secondary to COVID-19: a systematic review \\ Enfermedad de Parkinson secundaria a COVID-19: una revisión sistemática
}

Recebido: 06/12/2021 | Revisado: 12/12/2021 | Aceito: 11/01/2022| Publicado: 13/01/2022

Moisés Hamoy

ORCID: https://orcid.org/0000-0002-2931-4324 Universidade Federal do Pará, Brasil

E-mail: hamoyufpa@gmail.com

Gabriel Hans Reis Braga

ORCID: https://orcid.org/0000-0002-9644-8250 Universidade Federal do Pará, Brasil

E-mail:gabrielhansreisbraga@gmail.com

Jones Vinicius Santana Chaves

ORCID: https://orcid.org/0000-0002-3312-1437 Universidade Federal do Pará, Brasil

E-mail:jonesvinicius2222@gmail.com

Ana Carolina Serrão Maia

ORCID: https://orcid.org/0000-0002-8407-9101 Universidade Federal do Pará, Brasil

E-mail:acarolinasmaia@gmail.com

Diego Arthur Castro Cabral

ORCID: https://orcid.org/0000-0002-7582-7251 Universidade Federal do Pará, Brasil

E-mail: diego.arthur.castro@gmail.com

Fernanda Myllena Sousa Campos

ORCID: https://orcid.org/0000-0003-1903-0958 Universidade Federal do Pará, Brasil E-mail: fernanda.campos@ics.ufpa.br

João Paulo do Vale Medeiros

ORCID: https://orcid.org/0000-0002-5110-2379 Universidade Federal do Pará, Brasil E-mail:jp.vale.medeiros@gmail.com

Leonardo Giovanni Castro Cabral ORCID: https://orcid.org/0000-0002-8814-9488 Universidade Federal do Pará, Brasil E-mail: leonardo.castro26@yahoo.com

Bruno Patricio dos Santos Oliveira ORCID: https://orcid.org/0000-0002-7446-6626 Universidade Federal do Pará, Brasil E-mail: bruno.patricio@ics.ufpa.br Joyce Pantoja Braga

ORCID: https://orcid.org/0000-0003-3191-0077 Universidade Federal do Pará, Brasil E-mail: joyce.braga@ics.ufpa.br

Vanessa Jóia de Mello

ORCID: https://orcid.org/0000-0003-0359-9760 Universidade Federal do Pará, Brasil

E-mail: vanessajoiafarmacia@gmail.com

Eric Homero Albuquerque Paschoal

ORCID: https://orcid.org/0000-0002-6995-8466

Universidade Federal do Pará, Brasil E-mail: ericpaschoal@gmail.com

\section{Resumo}

Objetivo: O presente trabalho teve como objetivo realizar uma revisão sistemática a respeito da Doença de Parkinson e sua relação com a COVID-19. Métodos: Utilizamos e adaptamos os critérios presentes no PRISMA (Preferred Reporting Items for Systematic reviews and Meta-Analyses) e objetivou a identificação de publicações revisadas por pares pertencentes a temática da doença de Parkinson relacionada à COVID-19. Resultados: A doença de Parkinson após a COVID-19 está presente em alguns casos e parece estar relacionada com mecanismos de hiperinflamação mediada pelo Sars-Cov-2, principalmente em pacientes abaixo dos 60 anos. Conclusão: A ocorrência da doença de Parkinson após uma infecção pelo novo coronavírus parece ser explicada pelos efeitos citolíticos do vírus, acúmulo de 
proteínas intracelulares, modificações epigenéticas e interações em vias inflamatórias que causam morte neuronal. A COVID-19 também está associada à piora dos sintomas da Doença de Parkinson previamente diagnosticada.

Palavras-chave: Doença de Parkinson; Infecções por coronavírus; Neurologia.

\begin{abstract}
Objective: This study aimed to carry out a systematic review of Parkinson's Disease and its relationship with COVID19. Methods: We used and adapted the criteria present in PRISMA (Preferred Reporting Items for Systematic Reviews and Meta-Analyses) and aimed to identify peer-reviewed publications on the topic of Parkinson's disease related to COVID-19. Results: Parkinson's disease after COVID-19 is present in some cases and seems to be related to mechanisms of hyperinflammation mediated by Sars-Cov-2, especially in patients under 60 years of age. Conclusion: The occurrence of Parkinson's disease after an infection with the new coronavirus seems to be explained by the cytolytic effects of the virus, accumulation of intracellular proteins, epigenetic modifications and interactions in inflammatory pathways that cause neuronal death. COVID-19 is also associated with worsening of the symptoms in people with previously diagnosed Parkinson's Disease.
\end{abstract}

Keywords: Parkinson's Disease; Coronavirus infections; Neurology.

\title{
Resumen
}

Objetivo: Este estudio tuvo como objetivo realizar una revisión sistemática de la Enfermedad de Parkinson y su relación con COVID-19. Métodos: Utilizamos y adaptamos los criterios presentes en PRISMA (Preferred Reporting Items for Systematic Reviews and Meta-Analysis) y buscamos identificar publicaciones revisadas por pares sobre el tema de la enfermedad de Parkinson relacionada con COVID-19. Resultados: La enfermedad de Parkinson posterior al COVID19 está presente en algunos casos y parece estar relacionada con mecanismos de hiperinflamación mediados por SarsCov-2, especialmente en pacientes menores de 60 años. Conclusión: La aparición de la enfermedad de Parkinson tras una infección por el nuevo coronavirus parece explicarse por los efectos citolíticos del virus, acumulación de proteínas intracelulares, modificaciones epigenéticas e interacciones en vías inflamatorias que provocan muerte neuronal. COVID-19 también se asocia con el empeoramiento de los síntomas de la enfermedad de Parkinson diagnosticada previamente.

Palabras clave: Enfermedad de Parkinson; Infecciones por coronavirus; Neurología.

\section{Introdução}

A pandemia causada pelo novo coronavírus (Sars-Cov-2) atingiu o mundo inteiro através da sua doença (COVID-19) que pode variar desde casos assintomáticos até quadros com risco à vida devido seu comprometimento multissistêmico.(Wiersinga et al., 2020) O encéfalo parece ter uma invasão viral, como alguns estudos post mortem revelam (Ding et al., 2004; Gu et al., 2005) mas o que a maioria dos estudos até o momento indicam é que mesmo com alta carga viral e reação inflamatória sistêmica grave, o mecanismo mais provável das manifestações neurológicas é a resposta inflamatória exacerbada.(Pilotto et al., 2020)

Algumas encefalites virais parecem estar ligadas diretamente à indução de doenças neurodegenerativas. (Jang et al., 2009) A ideia de vírus como causa da DP ou de parkinsonismo secundário já é descrita desde os casos de parkinsonismo pósencefalítico observados durante a pandemia da gripe espanhola em 1918 (Dickman, 2001; Sulzer et al., 2020). Outros vírus também são associados ao parkinsonismo como a influenza, o Coxsackie, o vírus da encefalite B japonesa, encefalite equina ocidental, herpes, HIV e, recentemente, há relatos de casos após a infecção pelo Sars-Cov-2 (Cohen et al., 2020; Merello et al., 2021; Pilotto et al., 2020). O presente estudo tem como objetivo sintetizar o conhecimento disponível sobre a relação entre a COVID-19 e a doença de Parkinson através de revisão sistemática.

\section{Metodologia}

\subsection{Métodos}

O presente trabalho utilizou e adaptou o sistema de critérios presentes no PRISMA (Preferred Reporting Items for Systematic reviews and Meta-Analyses) (Moher et al., 2010) e objetivou a identificação de todas as publicações revisadas por pares pertencentes a temática da doença de Parkinson relacionada à COVID-19. 


\subsection{Estratégia de pesquisa}

Uma pesquisa eletrônica foi elaborada usando os seguintes bancos de dados: PubMed e Cochrane Library para estudos publicados a partir de janeiro de 2011. As palavras-chave usadas para pesquisas nesses bancos de dados incluíram o seguinte: Doença de Parkinson, Parkinsonismo e COVID-19. Além disso, foram revisados manualmente as referências dos estudos incluídos, a fim de identificar artigos potencialmente elegíveis. (Texakalidis et al., 2018, 2019).

\subsubsection{Critérios de inclusão e exclusão}

Uma Os artigos foram avaliados e selecionados pelo seu título, resumo e leitura do texto completo para extração dos dados. Foram adicionados trabalhos avaliados por pares, escritos na língua inglesa e disponíveis nas plataformas utilizadas para coleta dos dados. Textos que não estavam escritos em inglês, pre-prints e sem avaliação por pares foram excluídos. Em casos de estudos duplicados, incluímos a publicação mais recente, a menos que os desfechos de interesse fossem relatados na versão mais antiga. (Texakalidis et al., 2018). Devido ao tamanho limitado das amostras incluídas no estudo, decidimos não realizar uma meta-análise dos dados aqui apresentados.

\subsubsection{Extração dos dados}

Todos os estudos foram sistematicamente revisados de forma independente por 2 revisores (DACC, FMSC) e quaisquer desvios dos critérios mencionados acima foram registrados. As discrepâncias entre os revisores foram resolvidas consultando o autor sênior (EHAP) (Texakalidis et al., 2019). Os dados extraídos incluíram título, ano de publicação, diagnóstico da DP ou Parkinsonismo, desenho do estudo, intervalo de tempo do diagnóstico e principais achados.

\section{Resultados}

Nossa pesquisa resultou em um total de 202 artigos oriundos das plataformas utilizadas. Após a seleção baseada em seus títulos e resumos, 07 artigos foram submetidos à avaliação de texto completo e atenderam aos critérios de elegibilidade predefinidos para inclusão nesta revisão (Figura 1).

Figura 1. Fluxo de trabalho PRISMA para seleção de artigos.
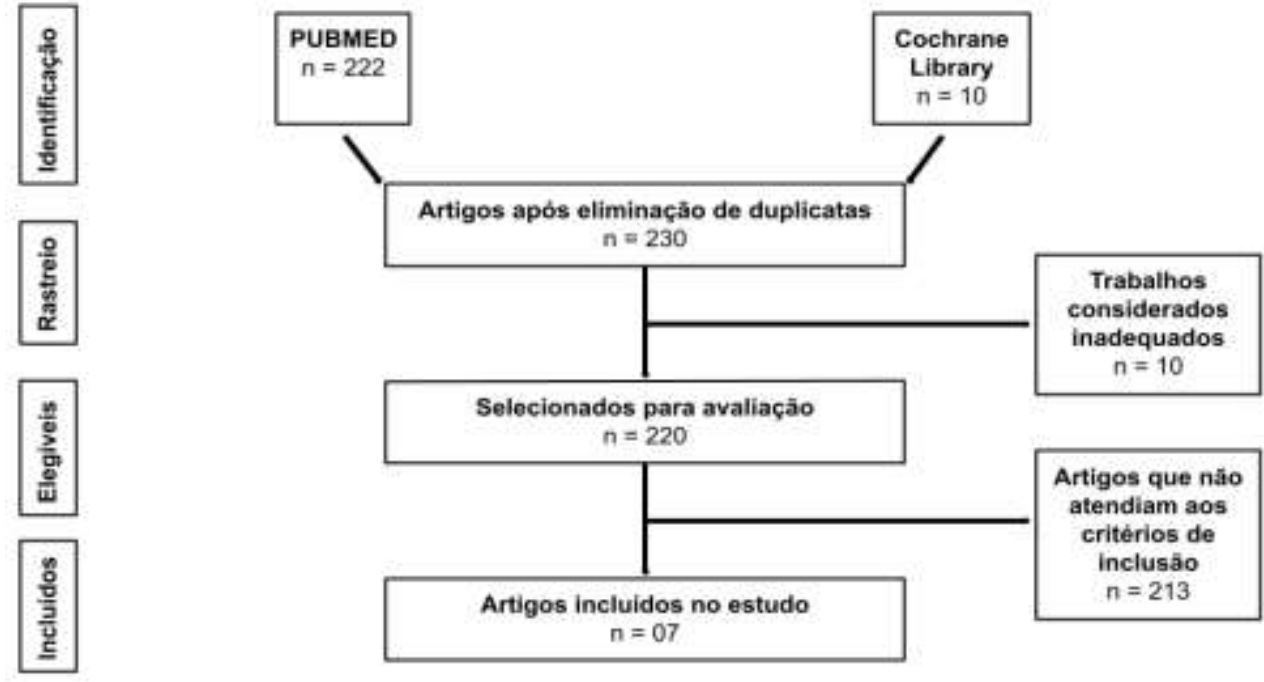

Fonte: Autores.

Os trabalhos selecionados foram organizados na Tabela 1 (Chaudhry et al., 2020; Cilia et al., 2020; Cohen et al., 2020). 
Research, Society and Development, v. 11, n. 1, e50711124397, 2022

(CC BY 4.0) | ISSN 2525-3409 | DOI: http://dx.doi.org/10.33448/rsd-v11i1.24397

Tabela 1 - Estudos selecionados para incluir a revisão sistemática.

\begin{tabular}{|c|c|c|c|c|c|}
\hline Título & Ano & Diagnóstico & Desenho do estudo & $\begin{array}{l}\text { Tempo de } \\
\text { diagnóstico }\end{array}$ & Principais achados \\
\hline $\begin{array}{l}\text { COVID-19 and Parkinson's Disease: Shared Inflammatory } \\
\text { Pathways Under Oxidative Stress } \uparrow^{16}\end{array}$ & 2020 & NA & $\begin{array}{l}\text { Estudo } \\
\text { experimental }\end{array}$ & NA & $\begin{array}{c}\text { O estresse oxidativo comum à COVID-19 e a DP leva a morte de neurônios } \\
\text { dopaminérgicos }\end{array}$ \\
\hline $\begin{array}{l}\text { A case of probable Parkinson's disease after SARS-CoV-2 } \\
\text { infection }^{12}\end{array}$ & 2020 & $\begin{array}{l}\text { Clínico e } \\
\text { imagem }\end{array}$ & Relato de caso & dois meses & Possível invasão do Sars-Cov-2 no encéfalo facilitando o desenvolvimento da DP \\
\hline $\begin{array}{l}\text { The Human Coronavirus Receptor ANPEP (CD13) Is } \\
\text { Overexpressed in Parkinson's Disease }{ }^{17}\end{array}$ & 2020 & NA & $\begin{array}{c}\text { Estudo } \\
\text { experimental }\end{array}$ & post mortem & $\begin{array}{l}\text { níveis elevados de ANPEP podem estar associados ao desenvolvimento de DP e, } \\
\text { possivelmente, por meio de seu papel na inflamação, contribuir para a gravidade da } \\
\text { infecção por SARS-Cov-2 }\end{array}$ \\
\hline $\begin{array}{l}\text { Acute hypokinetic-rigid syndrome following SARS-CoV-2 } \\
\text { infection }{ }^{18}\end{array}$ & 2020 & $\begin{array}{l}\text { Clínico e } \\
\text { imagem }\end{array}$ & Relato de caso & um mês & $\begin{array}{l}\text { fornece evidências de neuroimagem clínica e funcional para apoiar que o SARS - CoV-2 } \\
\text { pode obter acesso ao SNC, afetando as estruturas do mesencéfalo e levando a sinais e } \\
\text { sintomas neurológicos. }\end{array}$ \\
\hline $\begin{array}{l}\text { Human coronaviruses in idiopathic Parkinson's disease: } \\
\text { Implications of SARS-CoV-2's modulation of the host's } \\
\text { transcriptome }^{19}\end{array}$ & 2021 & NA & $\begin{array}{l}\text { Estudo } \\
\text { experimental }\end{array}$ & NA & $\begin{array}{l}\text { este estudo transcriptômico comparativo descreveu a potencial contribuição dos } \\
\text { coronavírus humanos na patogênese da doença de Parkinson idiopática }\end{array}$ \\
\hline $\begin{array}{l}\text { Coronavirus Disease } 2019 \text { and Parkinsonism: A Non-post- } \\
\text { encephalitic Case }{ }^{20}\end{array}$ & 2020 & $\begin{array}{l}\text { Clínico e } \\
\text { imagem }\end{array}$ & Relato de caso & duas semanas & $\begin{array}{l}\text { Relatam um caso de parkinsonismo responsivo à levodopa provavelmente causado por } \\
\text { infecção direta do SARS - CoV - } 2 \text {, ampliando o espectro clínico da doença. }\end{array}$ \\
\hline $\begin{array}{l}\text { Effects of COVID-19 on Parkinson's Disease Clinical } \\
\text { Features: A Community-Based Case-Control Study }{ }^{21}\end{array}$ & 2020 & Clínico & $\begin{array}{l}\text { Estudo de caso } \\
\text { controle baseado } \\
\text { na comunidade }\end{array}$ & 6.3 anos & $\begin{array}{l}\text { Pacientes com DP podem apresentar piora substancial dos sintomas motores e não } \\
\text { motores durante a doença COVID - } 19 \text { leve a moderada, independentemente da idade e } \\
\text { da duração da doença. }\end{array}$ \\
\hline
\end{tabular}

Fonte: Autores. 


\subsection{Características demográficas}

Os pacientes com critérios clínicos para o diagnóstico de Parkinson após a infecção pelo novo coronavírus (confirmada por RT-PCR) eram pacientes fora da faixa etária epidemiológica, todos com 60 anos ou menos. Nenhum paciente possuía histórico da doença de Parkinson na família e a maioria foi testada geneticamente para rastreamento dos principais genes relacionados a DP, com a testagem sendo negativa em todos os casos. Também não houve história de constipação crônica, distúrbios do sono REM e depressão. A maior incidência de casos acometeu o sexo masculino (Cohen et al., 2020; Faber et al., 2020; Méndez-Guerrero et al., 2020).

\subsection{Quadro clínico e gravidade da infecção pelo Sars-Cov-2}

A maioria dos pacientes apresentou sintomas de vias aéreas superiores como manifestações iniciais da COVID-19. Dos três relatos de casos incluídos, dois pacientes não precisaram de hospitalização e não tiveram quadros graves e um necessitou de cuidados intensivos em unidade de terapia intensiva. Outros sinais e sintomas incluem: dispnéia, mialgia, dor torácica, febre, fadiga, diarreia, anosmia e hipogeusia (Cohen et al., 2020; Faber et al., 2020; Méndez-Guerrero et al., 2020).

\subsection{Tempo de início das manifestações neurológicas}

O tempo de início dos primeiros sinais e sintomas de comprometimento neurológico desses pacientes se estabeleceu por volta de 17 dias após o início dos sintomas da infecção. Em geral, os primeiros sintomas não foram relacionados à DP em si, mas outros achados como: hemiplegias, hemiparesias, mioclonias, ptose palpebral e comprometimento do tato fino. Outros achados nos pacientes mais graves foram a presença de crises convulsivas e rebaixamento do nível de consciência (Cohen et al., 2020; Faber et al., 2020; Méndez-Guerrero et al., 2020).

\subsection{Principais achados clínicos da Doença de Parkinson}

Os principais achados do exame clínico dos pacientes foi a presença de bradicinesia, tremor em repouso, hipomimia, fala hipofônica, sinal da roda denteada em um ou mais membros, lentidão na marcha, rigidez, marcha com redução do balanço dos braços, en bloc turning, diminuição da passada durante a caminhada, hiposmia e fácie em máscara. Nenhum deles apresentou déficit cognitivo ou distúrbios na atenção, memória, linguagem e/ou déficits sensitivos (Cohen et al., 2020; Faber et al., 2020; Méndez-Guerrero et al., 2020).

\subsection{Exames complementares}

Os exames de neuroimagem convencionais (tomografia computadorizada de encéfalo, ressonância magnética nuclear de encéfalo e angiotomografia computadorizada de encéfalo) não apresentaram alterações intracranianas nos pacientes estudados (Cohen et al., 2020; Faber et al., 2020; Méndez-Guerrero et al., 2020). O eletroencefalograma também não possuía alterações. O único exame que demonstrou alterações foi a Tomografia por emissão de pósitrons que demonstrou uma redução na absorção de 18F-FDOPA no putamen e diminuição da densidade do transportador de dopamina no putâmen esquerdo (Cohen et al., 2020; Faber et al., 2020; Méndez-Guerrero et al., 2020).

Os exames laboratoriais não possuíam nenhuma alteração significativa que não fosse correlacionado ao quadro da infecção sistêmica. A análise do líquido cefalorraquidiano também não demonstrou a presença do vírus ou de anticorpos contra ele (Cohen et al., 2020; Faber et al., 2020; Méndez-Guerrero et al., 2020). 


\subsection{Tratamento}

De posse do diagnóstico clínico da doença, o tratamento farmacológico foi estabelecido para a grande maioria dos pacientes. Apenas um caso não precisou de farmacoterapia, pois apresentou melhora significativa no tremor, rigidez e bradicinesia sem qualquer tratamento específico (Méndez-Guerrero et al., 2020) A farmacoterapia de escolha foi realizada com a administração de Levodopa, Biperideno e Benserazida (Cohen et al., 2020; Faber et al., 2020).

No caso de pacientes que já tinham diagnóstico da DP, foi observada uma maior necessidade de aumento na dosagem da terapia dopaminérgica para pacientes infectados em relação a outros pacientes que não tiveram a COVID-19 (Cilia et al., 2020).

\subsection{Efeitos da COVID-19 em pacientes com diagnóstico prévio da doença de Parkinson}

Para os pacientes que já tinham diagnóstico prévio da DP e foram acometidos pela COVID-19 houve piora significativa dos escores de Impressão clínica do índice de gravidade para doença de Parkinson (CISI-PD) (Martínez-Martín et al., 2006), na Escala de avaliação da DP da Sociedade de Distúrbios do Movimento (MDS-UPDRS) Partes II e IV (MDS-UPDRS Parte II) (Goetz et al., 2008), no escore total da Versão italiana da Escala de Sintomas Não Motores (NMSS) (Cova et al., 2017) e sub pontuação do domínio urinário. A análise de caso-controle entre grupos revelou adicionalmente maior deficiência motora (no CISI-PD), flutuações motoras (no MDS-UPDRS Parte IV) e queixas não motoras. O envolvimento das funções cognitivas foi irrelevante (sem alteração no CISI - PD), e as funções cardiovasculares e sexuais autonômicas permaneceram inalteradas (Cilia et al., 2020).

\subsection{Papel do estresse oxidativo e de fatores genéticos}

A tempestade inflamatória encontrada na COVID-19 envolve a ativação das células endoteliais da vasculatura pulmonar levando ao desenvolvimento de hipóxia (Dong et al., 2020; Huang et al., 2020) A hipóxia respiratória observada em pacientes com COVID-19 gera estresse oxidativo no cérebro através da produção de espécies reativas de oxigênio (EROS) (CoimbraCosta et al., 2017). Níveis elevados de EROS causam desequilíbrio redox, aumentam os produtos de peroxidação lipídica e induzem distúrbios mitocondriais causando ativação das pró-caspases, do fator de iniciação de apoptose e do citocromo c, que induzem a morte celular por apoptose (Görlach et al., 2015; Hernansanz-Agustín et al., 2014; Niizuma et al., 2009). O estresse oxidativo causado pelo aumento da produção de EROS após a hipóxia contribui para a morte de neurônios contendo dopamina (NCD), podendo levar ao desenvolvimento da DP (Andrzejewski et al., 2020; Deumens et al., 2002; Prieto-Lloret et al., 2007).

Existe uma semelhança entre as proteínas Nsp3 do novo coronavírus e a PARP14 humana. A PARP14 está envolvida na via de sinalização do receptor de interferons (IFN), que contribui para a formação de fatores na cascata inflamatória que se translocam para o núcleo e afetam a expressão gênica (Chaudhry et al., 2020; Iwata et al., 2016) A PARP14 impede a fosforilação do transdutor de sinal e ativador da transcrição 1 (STAT1), o que aumenta a ativação de NFkB e, como consequência, regula negativamente as respostas desencadeadas pelos IFNs. (Claverie, 2020) Considerando que a Nsp3 compartilha similaridade com o PARP14, foi sugerido que os dois possuem conformação e compatibilidade semelhantes para a remoção e adição de ADPribose da STAT1. Assim, em uma célula ativada por IFN, o Nsp3 viral expresso irá neutralizar a mono-ADP-ribosilação da STAT1 e, portanto, regular positivamente a fosforilação da STAT1. Isto dá suporte ao papel potencial da Nsp3 na promoção da fosforilação da STAT1 em resposta à estimulação de IFN- $\gamma$ (Chaudhry et al., 2020; Iwata et al., 2016; Y. Zhang et al., 2015). Esta expressão prolongada pode gerar as várias consequências correspondentes à gravidade da COVID-19, incluindo inflamação intensificada e a tempestade de citocinas, conforme descrito por Claverie (Claverie, 2020).

$\mathrm{O}$ estresse oxidativo causado pela hipóxia respiratória também ativa o NFKB e tem um impacto negativo na função da proteína de Parkinson 7-PARK7 (DJ-1). A perda da DJ-1 leva ao aumento da fosforilação de STAT1 e mediadores inflamatórios 
regulados positivamente em resposta ao IFN- $\gamma$, semelhante à infecção por SARS-CoV-2 o que pode aumentar o risco da DP (Kim et al., 2013).

O mecanismo genético pelo qual o SARS-CoV-2 pode contribuir especificamente para o parkinsonismo e a DP provavelmente envolve a introdução ou ativação de mutações em genes associados à disproteostase e, especificamente, autofagia - mecanismos utilizados pelos betacoronavírus como uma estratégia de imunoevasão e latência (Vavougios, 2020, 2021).

\subsection{Prováveis vias de invasão do novo coronavírus ao encéfalo}

Os coronavírus podem invadir o sistema nervoso central de várias maneiras.Uma delas é através de sua inoculação intranasal, onde ele pode migrar transneuronalmente para estruturas de primeira e segunda ordem conectadas ao bulbo olfatório (Jang et al., 2012; Xu et al., 2005). Sendo assim, é provável que o Sars-CoV-2 possa invadir as populações neuronais do bulbo olfatório, seguindo para o diencéfalo e, eventualmente alcançando o tronco cerebral (Rábano-Suárez et al., 2020). Estudos em animais demonstraram que a invasão dos vírus induz uma resposta celular mínima e não é detectada através de estudos do LCR (Netland et al., 2008).

Outras evidências sugerem que o Sars-CoV-2 entra em organóides do cérebro humano 2 dias após a exposição e tem como alvo preferencial os neurônios desses organóides. A exposição ao SARS-CoV-2 está associada à distribuição alterada da proteína Tau dos axônios ao soma, hiperfosforilação e morte neuronal (Ramani et al., 2020).

Ao que parece o novo coronavírus também invade o encéfalo através da via hematogênica. Nela, os vírus obtêm acesso infectando células endoteliais da barreira hematoencefálica, células epiteliais da barreira hemato-cefalorraquidiana no plexo coróide ou usam células inflamatórias como cavalos de Tróia para obter acesso ao SNC. Essa evidência é reforçada pela observação de partículas virais do Sars-Cov-2 no endotélio capilar encefálico (Desforges et al., 2019; Paniz-Mondolfi et al., 2020).

\subsection{Mecanismos envolvidos nos distúrbios neurológicos: Doença de Parkinson}

A DP pode ser induzida através de infecções virais por diversos mecanismos. Alguns modelos animais propuseram uma disfunção dos neurônios nigroestriatais, ou até mesmo, efeitos citolíticos diretos (Jang et al., 2012; Netland et al., 2008) Susceptibilidades genéticas e ativação microglial, também podem facilitar o acúmulo de proteínas e a neurodegeneração (Sadasivan et al., 2015). Mas o que parece ser mais plausível seria uma combinação do estresse oxidativo e uma inibição das respostas neuroprotetoras podendo levar à morte neuronal (Sulzer, 2007).

\section{Discussão}

A doença de Parkinson é a segunda doença neurodegenerativa mais comum e tende a aumentar sua incidência com o envelhecimento crescente da população no mundo (Wiersinga et al., 2020). No último ano tivemos diversos estudos demonstrando uma relação direta entre a COVID-19 e o aparecimento da DP em pacientes sem diagnóstico de doenças neurológicas (Cohen et al., 2020; Faber et al., 2020; Méndez-Guerrero et al., 2020). O desenvolvimento da DP não parece estar relacionado à gravidade da infecção e não tem um prognóstico bem estabelecido, mas a maioria dos casos evolui com a necessidade de estabelecimento de uma farmacoterapia antiparkinsoniana tendo apenas um caso descrito com melhora dos sinais e sintomas do Parkinsonismo a despeito de quaisquer medidas terapêuticas (Méndez-Guerrero et al., 2020).

O intervalo de tempo entre as manifestações da COVID-19 e o aparecimento dos primeiros sinais e sintomas de distúrbios do movimento dura em torno de 2 semanas. Entre os principais achados clínicos podemos destacar a bradicinesia, tremor em repouso, diminuição da tonalidade da voz, lentidão da marcha e fácies em máscara. Esses achados são encontrados 
em pacientes abaixo dos 60 anos, sem histórico da doença de Parkinson na família e sem a presença de genes que estão relacionados ao desenvolvimento da DP (Cohen et al., 2020; Faber et al., 2020; Méndez-Guerrero et al., 2020). Ao analisarmos tais achados de desenvolvimento de distúrbios motores em pacientes jovens logo após uma infecção viral, fica muito sugestiva a relação causal entre a COVID-19 e o desenvolvimento da DP, como já foi estabelecido em estudos prévios (Jang et al., 2009; Takahashi \& Yamada, 1999).

Os exames de imagem mais comuns não apresentam alteração do parênquima encefálico. No entanto, esses métodos não são adequados para se avaliar alterações presentes na doença de Parkinson (Pagano et al., 2016). As alterações são melhor avaliadas quando utilizamos métodos de imagem mais sofisticados como a tomografia por emissão de pósitrons e a tomografia computadorizada por emissão de fóton único (Politis, 2014). Vale ressaltar que o novo coronavírus e seus anticorpos não foram encontrados nas análises do LCR realizadas durante os estudos analisados (Cohen et al., 2020; Faber et al., 2020; MéndezGuerrero et al., 2020). Uma das possíveis explicações para esse fato é que o vírus deve causar pouca inflamação e, consequentemente, pouco recrutamento de células inflamatórias, o que causa a sua não detecção no LCR (Netland et al., 2008). No entanto, estruturas virais e seu material genético já foram encontrados em análises ultraestruturais através da microscopia eletrônica de transmissão de seções em neurônios e células endoteliais de dois pacientes com o diagnóstico de COVID-19 (PanizMondolfi et al., 2020).

O Sars-Cov-2 provavelmente possui diversos mecanismos de invasão do SNC. Muitos deles são hipotetizados a partir de analogias realizadas com outros coronavírus e de estudos em animais, como é o caso da transmissão retrógrada a partir do nervo olfatório através dos núcleos do SNC, chegando ao tálamo e tronco cerebral (Netland et al., 2008). O coronavírus também pode invadir o encéfalo através da via hematogênica ou linfática (Ding et al., 2004; Wiersinga et al., 2020). Uma outra provável maneira do novo coronavírus ter acesso ao SNC é através da quebra da barreira hematoencefálica no momento da tempestade de citocinas da COVID-19 (Muldoon et al., 2013; Obermeier et al., 2013) A entrada do vírus no encéfalo parece estar relacionado à quantidade de receptores ACE2 no tecido cerebral (Netland et al., 2008).

Existe um número elevado de evidências que dão suporte às potenciais relações existentes entre a COVID-19 e a gênese de doenças neurodegenerativas como o Parkinson (Sulzer et al., 2020). Os neurônios dopaminérgicos são seletivamente vulneráveis por causa de suas propriedades intrínsecas que incluem altas demandas bioenergéticas de axônios altamente arborizados e proteostase prejudicada resultante do grande tamanho dos axônios que podem promover agregação de $\alpha$-sinucleína e resultar em vulnerabilidade seletiva a fatores autônomos não celulares que promovam a propagação de $\alpha$-sinucleína, como a neuroinflamação e as neurotoxinas ambientais (Pavel et al., 2020).

A proteína $\alpha$-sinucleína parece ter um papel antiviral dentro dos neurônios, desta forma o acúmulo desta proteína (que pode ter função antiviral) após a infecção por SARS-CoV-2 pode agravar a vulnerabilidade autônoma da célula pré-existente e levar à propagação de $\alpha$-sinucleína e neurodegeneração generalizada (Pavel et al., 2020). A infecção pelo novo coronavírus também pode interferir na depuração da $\alpha$-sinucleína, bem como outros vírus neurotrópicos o fazem (Marreiros et al., 2020).

O estresse bioenergético causado pela COVID-19 pode ser intransponível para certas populações neuronais. Os neurônios dopaminérgicos nigroestriatais necessitam de altas demandas de energia celular para alimentar a fosforilação oxidativa basal elevada em suas mitocôndrias, sua alta densidade terminal de axônios e sua extensa arborização axonal. Considerando essa grande demanda metabólica, se as reservas de energia celular não estiverem disponíveis, o estresse celular da infecção pela COVID-19 pode levar esses neurônios vulneráveis além do limiar da neurodegeneração (Pavel et al., 2020).

Além dos mecanismos supracitados, outras alterações estruturais como as lesões hipóxicas e neuroinflamação excessiva no encéfalo podem causar a gênese da DP (Haddadi et al., 2020). Também pode haver o aparecimento da doença de Parkinson previamentes instalada, mas, assintomática, ou a possibilidade do desenvolvimento da doença de Parkinson em longo prazo em indivíduos com susceptibilidade genética (Merello et al., 2021). Desta maneira, as proteínas como a Nsp3 do novo coronavírus 
e as alterações genéticas como a disproteostase também podem contribuir para o estabelecimento do quadro neurodegenerativo após a COVID-19.

As manifestações neurológicas da COVID-19 costumam ser variadas e se encontram dentro de um espectro de apresentações. No entanto, quando observamos os pacientes que já possuem a DP notamos que eles podem apresentar piora substancial dos sintomas motores e não motores durante a infecção, independentemente da idade, gravidade e da duração da doença (Cilia et al., 2020). Também foi descrito que os pacientes portadores da DP possuíam taxas de letalidade da COVID-19 variando de $0 \%$ a $40 \%$, e essas taxas se mantém elevadas entre os pacientes parkinsonianos independentemente da idade, sexo e raça (Q. Zhang et al., 2020). No entanto, a prevalência da doença nos pacientes com DP, não difere da prevalência da COVID19 na população geral (Artusi et al., 2020).

Embora o parkinsonismo agudo associado à COVID-19 pareça ser raro, a disseminação do novo coronavírus na sociedade pode levar a uma alta proporção de pessoas predispostas a desenvolver a DP mais tardiamente, especialmente porque serão afetados pelos processos de envelhecimento (Brundin et al., 2020). O mundo foi pego de surpresa pela pandemia da COVID-19, mas nós estaremos prontos para a próxima onda de sequelas neurológicas induzidas pela COVID-19, incluindo a doença de Parkinson? (Beauchamp et al., 2020).

\section{Conclusão}

A doença de Parkinson diagnosticada após a infecção pelo novo coronavírus pode ser explicada pelos efeitos citolíticos do vírus, bem como o acúmulo de proteínas intracelulares, modificações epigenéticas e interações em vias inflamatórias que modulam o organismo para uma resposta inflamatória exacerbada causando morte neuronal. A COVID-19 também está associada à piora dos sintomas da Doença de Parkinson previamente diagnosticada. Sendo assim, a DP após infecção pelo novo coronavírus deve ser foco da pesquisa neurológica, pois podemos estar diante de um expressivo aumento na incidência dos distúrbios do movimento nos anos pós pandemia.

\section{Referências}

Andrzejewski, K., Jampolska, M., Zaremba, M., Joniec-Maciejak, I., Boguszewski, P. M., \& Kaczyńska, K. (2020). Respiratory pattern and phrenic and hypoglossal nerve activity during normoxia and hypoxia in 6-OHDA-induced bilateral model of Parkinson's disease. The Journal of Physiological Sciences, 70(1), 16. https://doi.org/10.1186/s12576-020-00743-4

Artusi, C. A., Romagnolo, A., Imbalzano, G., Marchet, A., Zibetti, M., Rizzone, M. G., \& Lopiano, L. (2020). COVID-19 in Parkinson's disease: Report on prevalence and outcome. Parkinsonism \& Related Disorders, 80, 7-9. https://doi.org/10.1016/j.parkreldis.2020.09.008

Beauchamp, L. C., Finkelstein, D. I., Bush, A. I., Evans, A. H., \& Barnham, K. J. (2020). Parkinsonism as a Third Wave of the COVID-19 Pandemic? Journal of Parkinson's Disease, 10(4), 1343-1353. https://doi.org/10.3233/JPD-202211

Brundin, P., Nath, A., \& Beckham, J. D. (2020). Is COVID-19 a Perfect Storm for Parkinson's Disease? Trends in Neurosciences, 43(12), 931-933. https://doi.org/10.1016/j.tins.2020.10.009

Chaudhry, Z., Klenja, D., Janjua, N., Cami-Kobeci, G., \& Ahmed, B. (2020). COVID-19 and Parkinson's Disease: Shared Inflammatory Pathways Under Oxidative Stress. Brain Sciences, 10(11), 807. https://doi.org/10.3390/brainsci10110807

Cilia, R., Bonvegna, S., Straccia, G., Andreasi, N. G., Elia, A. E., Romito, L. M., Devigili, G., Cereda, E., \& Eleopra, R. (2020). Effects of <scp>COVID</scp> -19 on Parkinson's Disease Clinical Features: A $<$ scp $>$ Community-Based Case-Control</scp $>$ Study. Movement Disorders, 35(8), 1287-1292. https://doi.org/10.1002/mds.28170

Claverie, J.-M. (2020). A Putative Role of de-Mono-ADP-Ribosylation of STAT1 by the SARS-CoV-2 Nsp3 Protein in the Cytokine Storm Syndrome of COVID-19. Viruses, 12(6), 646. https://doi.org/10.3390/v12060646

Cohen, M. E., Eichel, R., Steiner-Birmanns, B., Janah, A., Ioshpa, M., Bar-Shalom, R., Paul, J. J., Gaber, H., Skrahina, V., Bornstein, N. M., \& Yahalom, G. (2020). A case of probable Parkinson's disease after SARS-CoV-2 infection. The Lancet Neurology, 19(10), 804-805. https://doi.org/10.1016/S14744422(20)30305-7 
Coimbra-Costa, D., Alva, N., Duran, M., Carbonell, T., \& Rama, R. (2017). Oxidative stress and apoptosis after acute respiratory hypoxia and reoxygenation in rat brain. Redox Biology, 12, 216-225. https://doi.org/10.1016/j.redox.2017.02.014

Cova, I., Battista, M. E. di, Vanacore, N., Papi, C. P., Alampi, G., Rubino, A., Valente, M., Meco, G., Contri, P., Pucchio, A. di, Lacorte, E., Priori, A., Mariani, C., \& Pomati, S. (2017). Validation of the Italian version of the Non Motor Symptoms Scale for Parkinson's disease. Parkinsonism \& Related Disorders, 34, 38-42. https://doi.org/10.1016/j.parkreldis.2016.10.020

Desforges, M., Coupanec, A. le, Dubeau, P., Bourgouin, A., Lajoie, L., Dubé, M., \& Talbot, P. J. (2019). Human Coronaviruses and Other Respiratory Viruses: Underestimated Opportunistic Pathogens of the Central Nervous System? Viruses, 12(1), 14. https://doi.org/10.3390/v12010014

Deumens, R., Blokland, A., \& Prickaerts, J. (2002). Modeling Parkinson's Disease in Rats: An Evaluation of 6-OHDA Lesions of the Nigrostriatal Pathway. Experimental Neurology, 175(2), 303-317. https://doi.org/10.1006/exnr.2002.7891

Dickman, M. S. (2001). von Economo Encephalitis. Archives of Neurology, 58(10), 1696. https://doi.org/10.1001/archneur.58.10.1696

Ding, Y., He, L., Zhang, Q., Huang, Z., Che, X., Hou, J., Wang, H., Shen, H., Qiu, L., Li, Z., Geng, J., Cai, J., Han, H., Li, X., Kang, W., Weng, D., Liang, P., \& Jiang, S. (2004). Organ distribution of severe acute respiratory syndrome(SARS) associated coronavirus(SARS-CoV) in SARS patients: implications for pathogenesis and virus transmission pathways. The Journal of Pathology, 203(2), 622-630. https://doi.org/10.1002/path.1560

Dong, S., Liu, P., Luo, Y., Cui, Y., Song, L., \& Chen, Y. (2020). Pathophysiology of SARS-CoV-2 infection in patients with intracerebral hemorrhage. Aging, 12(13), 13791-13802. https://doi.org/10.18632/aging.103511

Faber, I., Brandão, P. R. P., Menegatti, F., Bispo, D. D. C., Maluf, F. B., \& Cardoso, F. (2020). Coronavirus Disease 2019 and Parkinsonism: A Non-postencephalitic Case. Movement Disorders, 35(10), 1721-1722. https://doi.org/10.1002/mds.28277

Goetz, C. G., Tilley, B. C., Shaftman, S. R., Stebbins, G. T., Fahn, S., Martinez-Martin, P., Poewe, W., Sampaio, C., Stern, M. B., Dodel, R., Dubois, B., Holloway, R., Jankovic, J., Kulisevsky, J., Lang, A. E., Lees, A., Leurgans, S., LeWitt, P. A., Nyenhuis, D., ... LaPelle, N. (2008). Movement Disorder Societysponsored revision of the Unified Parkinson's Disease Rating Scale (MDS-UPDRS): Scale presentation and clinimetric testing results. Movement Disorders, 23(15), 2129-2170. https://doi.org/10.1002/mds.22340

Görlach, A., Dimova, E. Y., Petry, A., Martínez-Ruiz, A., Hernansanz-Agustín, P., Rolo, A. P., Palmeira, C. M., \& Kietzmann, T. (2015). Reactive oxygen species, nutrition, hypoxia and diseases: Problems solved? Redox Biology, 6, 372-385. https://doi.org/10.1016/j.redox.2015.08.016

Gu, J., Gong, E., Zhang, B., Zheng, J., Gao, Z., Zhong, Y., Zou, W., Zhan, J., Wang, S., Xie, Z., Zhuang, H., Wu, B., Zhong, H., Shao, H., Fang, W., Gao, D., Pei, F., Li, X., He, Z., ... Leong, A. S.-Y. (2005). Multiple organ infection and the pathogenesis of SARS. Journal of Experimental Medicine, 202(3), 415-424. https://doi.org/10.1084/jem.20050828

Haddadi, K., Ghasemian, R., \& Shafizad, M. (2020). Basal Ganglia Involvement and Altered Mental Status: A Unique Neurological Manifestation of Coronavirus Disease 2019. Cureus. https://doi.org/10.7759/cureus.7869

Hernansanz-Agustín, P., Izquierdo-Álvarez, A., Sánchez-Gómez, F. J., Ramos, E., Villa-Piña, T., Lamas, S., Bogdanova, A., \& Martínez-Ruiz, A. (2014). Acute hypoxia produces a superoxide burst in cells. Free Radical Biology and Medicine, 71, 146-156. https://doi.org/10.1016/j.freeradbiomed.2014.03.011

Huang, C., Wang, Y., Li, X., Ren, L., Zhao, J., Hu, Y., Zhang, L., Fan, G., Xu, J., Gu, X., Cheng, Z., Yu, T., Xia, J., Wei, Y., Wu, W., Xie, X., Yin, W., Li, H., Liu, M., ... Cao, B. (2020). Clinical features of patients infected with 2019 novel coronavirus in Wuhan, China. The Lancet, 395(10223), 497-506. https://doi.org/10.1016/S0140-6736(20)30183-5

Iwata, H., Goettsch, C., Sharma, A., Ricchiuto, P., Goh, W. W. bin, Halu, A., Yamada, I., Yoshida, H., Hara, T., Wei, M., Inoue, N., Fukuda, D., Mojcher, A., Mattson, P. C., Barabási, A.-L., Boothby, M., Aikawa, E., Singh, S. A., \& Aikawa, M. (2016). PARP9 and PARP14 cross-regulate macrophage activation via STAT1 ADP-ribosylation. Nature Communications, 7(1), 12849. https://doi.org/10.1038/ncomms12849

Jang, H., Boltz, D. A., Webster, R. G., \& Smeyne, R. J. (2009). Viral parkinsonism. Biochimica et Biophysica Acta (BBA) - Molecular Basis of Disease, 1792(7), 714-721. https://doi.org/10.1016/j.bbadis.2008.08.001

Jang, H., Boltz, D., McClaren, J., Pani, A. K., Smeyne, M., Korff, A., Webster, R., \& Smeyne, R. J. (2012). Inflammatory Effects of Highly Pathogenic H5N1 Influenza Virus Infection in the CNS of Mice. Journal of Neuroscience, 32(5), 1545-1559. https://doi.org/10.1523/JNEUROSCI.5123-11.2012

Kim, J., Choi, D., Jeong, H., Kim, J., Kim, D. W., Choi, S. Y., Park, S.-M., Suh, Y. H., Jou, I., \& Joe, E.-H. (2013). DJ-1 facilitates the interaction between STAT1 and its phosphatase, SHP-1, in brain microglia and astrocytes: A novel anti-inflammatory function of DJ-1. Neurobiology of Disease, 60, 1-10. https://doi.org/10.1016/j.nbd.2013.08.007

Marreiros, R., Müller-Schiffmann, A., Trossbach, S. v, Prikulis, I., Hänsch, S., Weidtkamp-Peters, S., Moreira, A. R., Sahu, S., Soloviev, I., Selvarajah, S., Lingappa, V. R., \& Korth, C. (2020). Disruption of cellular proteostasis by H1N1 influenza A virus causes $\alpha$-synuclein aggregation. Proceedings of the National Academy of Sciences, 117(12), 6741-6751. https://doi.org/10.1073/pnas.1906466117

Martínez-Martín, P., Forjaz, M. J., Cubo, E., Frades, B., \& de Pedro Cuesta, J. (2006). Global versus factor-related impression of severity in Parkinson's disease: A new clinimetric index (CISI-PD). Movement Disorders, 21(2), 208-214. https://doi.org/10.1002/mds.20697

Méndez-Guerrero, A., Laespada-García, M. I., Gómez-Grande, A., Ruiz-Ortiz, M., Blanco-Palmero, V. A., Azcarate-Diaz, F. J., Rábano-Suárez, P., ÁlvarezTorres, E., de Fuenmayor-Fernández de la Hoz, C. P., Pérez, D. V., Rodríguez-Montalbán, R., Pérez-Rivilla, A., Catalán, J. S., Ramos-González, A., \& de la 
Aleja, J. G. (2020). Acute hypokinetic-rigid syndrome following SARS-CoV-2 infection. Neurology, 95(15), e2109-e2118. https://doi.org/10.1212/WNL.0000000000010282

Merello, M., Bhatia, K. P., \& Obeso, J. A. (2021). SARS-CoV-2 and the risk of Parkinson's disease: facts and fantasy. The Lancet Neurology, 20(2), 94-95. https://doi.org/10.1016/S1474-4422(20)30442-7

Moher, D., Liberati, A., Tetzlaff, J., \& Altman, D. G. (2010). Preferred reporting items for systematic reviews and meta-analyses: The PRISMA statement. International Journal of Surgery, 8(5), 336-341. https://doi.org/10.1016/j.ijsu.2010.02.007

Muldoon, L. L., Alvarez, J. I., Begley, D. J., Boado, R. J., del Zoppo, G. J., Doolittle, N. D., Engelhardt, B., Hallenbeck, J. M., Lonser, R. R., Ohlfest, J. R., Prat, A., Scarpa, M., Smeyne, R. J., Drewes, L. R., \& Neuwelt, E. A. (2013). Immunologic Privilege in the Central Nervous System and the Blood-Brain Barrier. Journal of Cerebral Blood Flow \& Metabolism, 33(1), 13-21. https://doi.org/10.1038/jcbfm.2012.153

Netland, J., Meyerholz, D. K., Moore, S., Cassell, M., \& Perlman, S. (2008). Severe Acute Respiratory Syndrome Coronavirus Infection Causes Neuronal Death in the Absence of Encephalitis in Mice Transgenic for Human ACE2. Journal of Virology, 82(15), 7264-7275. https://doi.org/10.1128/JVI.00737-08

Niizuma, K., Endo, H., \& Chan, P. H. (2009). Oxidative stress and mitochondrial dysfunction as determinants of ischemic neuronal death and survival. Journal of Neurochemistry, 109, 133-138. https://doi.org/10.1111/j.1471-4159.2009.05897.x

Obermeier, B., Daneman, R., \& Ransohoff, R. M. (2013). Development, maintenance and disruption of the blood-brain barrier. Nature Medicine, 19(12), 15841596. https://doi.org/10.1038/nm.3407

Pagano, G., Niccolini, F., \& Politis, M. (2016). Imaging in Parkinson's disease. Clinical Medicine, 16(4), 371-375. https://doi.org/10.7861/clinmedicine.16-4371

Paniz-Mondolfi, A., Bryce, C., Grimes, Z., Gordon, R. E., Reidy, J., Lednicky, J., Sordillo, E. M., \& Fowkes, M. (2020). Central nervous system involvement by severe acute respiratory syndrome coronavirus-2 (SARS-CoV-2). Journal of Medical Virology, 92(7), 699-702. https://doi.org/10.1002/jmv.25915

Pavel, A., Murray, D. K., \& Stoessl, A. J. (2020). COVID-19 and selective vulnerability to Parkinson's disease. The Lancet Neurology, 19(9), 719. https://doi.org/10.1016/S1474-4422(20)30269-6

Pilotto, A., Odolini, S., Masciocchi, S., Comelli, A., Volonghi, I., Gazzina, S., Nocivelli, S., Pezzini, A., Focà, E., Caruso, A., Leonardi, M., Pasolini, M. P., Gasparotti, R., Castelli, F., Ashton, N. J., Blennow, K., Zetterberg, H., \& Padovani, A. (2020). Steroid-Responsive Encephalitis in Coronavirus Disease 2019. Annals of Neurology, 88(2), 423-427. https://doi.org/10.1002/ana.25783

Politis, M. (2014). Neuroimaging in Parkinson disease: from research setting to clinical practice. Nature Reviews Neurology, 10(12), 708-722. https://doi.org/10.1038/nrneurol.2014.205

Prieto-Lloret, J., Donnelly, D. F., Rico, A. J., Moratalla, R., González, C., \& Rigual, R. J. (2007). Hypoxia transduction by carotid body chemoreceptors in mice lacking dopamine D 2 receptors. Journal of Applied Physiology, 103(4), 1269-1275. https://doi.org/10.1152/japplphysiol.00391.2007

Rábano-Suárez, P., Bermejo-Guerrero, L., Méndez-Guerrero, A., Parra-Serrano, J., Toledo-Alfocea, D., Sánchez-Tejerina, D., Santos-Fernández, T., FolgueiraLópez, M. D., Gutiérrez-Gutiérrez, J., Ayuso-García, B., de la Aleja, J. G., \& Benito-León, J. (2020). Generalized myoclonus in COVID-19. Neurology, 95(6), e767-e772. https://doi.org/10.1212/WNL.0000000000009829

Ramani, A., Müller, L., Ostermann, P. N., Gabriel, E., Abida-Islam, P., Müller-Schiffmann, A., Mariappan, A., Goureau, O., Gruell, H., Walker, A., Andrée, M., Hauka, S., Houwaart, T., Dilthey, A., Wohlgemuth, K., Omran, H., Klein, F., Wieczorek, D., Adams, O., ... Gopalakrishnan, J. (2020). <scp>SARS</scp> -CoV-2 targets neurons of 3D human brain organoids. The EMBO Journal, 39(20). https://doi.org/10.15252/embj.2020106230

Sadasivan, S., Zanin, M., O’Brien, K., Schultz-Cherry, S., \& Smeyne, R. J. (2015). Induction of Microglia Activation after Infection with the Non-Neurotropic A/CA/04/2009 H1N1 Influenza Virus. PLOS ONE, 10(4), e0124047. https://doi.org/10.1371/journal.pone.0124047

Sulzer, D. (2007). Multiple hit hypotheses for dopamine neuron loss in Parkinson's disease. Trends in Neurosciences, 30(5), 244-250. https://doi.org/10.1016/j.tins.2007.03.009

Sulzer, D., Antonini, A., Leta, V., Nordvig, A., Smeyne, R. J., Goldman, J. E., Al-Dalahmah, O., Zecca, L., Sette, A., Bubacco, L., Meucci, O., Moro, E., Harms, A. S., Xu, Y., Fahn, S., \& Chaudhuri, K. R. (2020). COVID-19 and possible links with Parkinson's disease and parkinsonism: from bench to bedside. Npj Parkinson's Disease, 6(1), 18. https://doi.org/10.1038/s41531-020-00123-0

Takahashi, M., \& Yamada, T. (1999). Viral etiology for Parkinson's disease-a possible role of influenza A virus infection. Japanese Journal of Infectious Diseases, 52(3), 89-98.

Texakalidis, P., Giannopoulos, S., Jonnalagadda, A. K., Kokkinidis, D. G., Machinis, T., Reavey-Cantwell, J., Armstrong, E. J., \& Jabbour, P. (2018). Carotid Artery Endarterectomy versus Carotid Artery Stenting for Restenosis After Carotid Artery Endarterectomy: A Systematic Review and Meta-Analysis. World Neurosurgery, 115, 421-429.e1. https://doi.org/10.1016/j.wneu.2018.02.196

Texakalidis, P., Lu, V. M., Yolcu, Y., Kerezoudis, P., Alvi, M. A., Parney, I. F., Fogelson, J. L., \& Bydon, M. (2019). Impact of Powdered Vancomycin on Preventing Surgical Site Infections in Neurosurgery: A Systematic Review and Meta-analysis. Neurosurgery, 84(3), 569-580. https://doi.org/10.1093/neuros/nyy288 
Research, Society and Development, v. 11, n. 1, e50711124397, 2022 (CC BY 4.0) | ISSN 2525-3409 | DOI: http://dx.doi.org/10.33448/rsd-v11i1.24397

Vavougios, G. D. (2020). A data-driven hypothesis on the epigenetic dysregulation of host metabolism by SARS coronaviral infection: Potential implications for the SARS-CoV-2 modus operandi. Medical Hypotheses, 140, 109759. https://doi.org/10.1016/j.mehy.2020.109759

Vavougios, G. D. (2021). Human coronaviruses in idiopathic Parkinson's disease: Implications of SARS-CoV-2's modulation of the host's transcriptome. Infection, Genetics and Evolution, 89, 104733. https://doi.org/10.1016/j.meegid.2021.104733

Wiersinga, W. J., Rhodes, A., Cheng, A. C., Peacock, S. J., \& Prescott, H. C. (2020). Pathophysiology, Transmission, Diagnosis, and Treatment of Coronavirus Disease 2019 (COVID-19). JAMA, 324(8), 782. https://doi.org/10.1001/jama.2020.12839

Xu, J., Zhong, S., Liu, J., Li, L., Li, Y., Wu, X., Li, Z., Deng, P., Zhang, J., Zhong, N., Ding, Y., \& Jiang, Y. (2005). Detection of Severe Acute Respiratory Syndrome Coronavirus in the Brain: Potential Role of the Chemokine Mig in Pathogenesis. Clinical Infectious Diseases, 41(8), 1089-1096. https://doi.org/10.1086/444461

Zhang, Q., Schultz, J. L., Aldridge, G. M., Simmering, J. E., \& Narayanan, N. S. (2020). <scp>Coronavirus Disease 201</scp> 9 Case Fatality and Parkinson's Disease. Movement Disorders, 35(11), 1914-1915. https://doi.org/10.1002/mds.28325

Zhang, Y., Mao, D., Roswit, W. T., Jin, X., Patel, A. C., Patel, D. A., Agapov, E., Wang, Z., Tidwell, R. M., Atkinson, J. J., Huang, G., McCarthy, R., Yu, J., Yun, N. E., Paessler, S., Lawson, T. G., Omattage, N. S., Brett, T. J., \& Holtzman, M. J. (2015). PARP9-DTX3L ubiquitin ligase targets host histone H2BJ and viral 3C protease to enhance interferon signaling and control viral infection. Nature Immunology, 16(12), 1215-1227. https://doi.org/10.1038/ni.3279 